\title{
Research Paper: Comparison of Inhibitory Control Skill in 6 to 8-Year-Old Children With Fluent Speech and Developmental Stuttering
}

\author{
Atefeh Rahmati $^{1}$ (D), Morteza Farazi ${ }^{*}$ (D), Farhad Choopanian ${ }^{1}$, Mahdi Tahamtan², Aliakbar Dashtelei $^{3}$, Hashem Farhangdoosth
}

1. Department of Speech Therapy, University of Social Welfare and Rehabilitation Sciences, Tehran, Iran.

2. Department of Communication Sciences and Disorders, College of Health and Human Services, Bowling Green State University, Bowling Green, Ohio, United States of America.

3. Department of Speech Therapy, School of Rehabilitation, Tehran University of Medical Sciences, Tehran, Iran.

\begin{tabular}{|c|c|}
\hline $\begin{array}{l}\text { Use your device to scan } \\
\text { and read the article online } \\
\square\end{array}$ & $\begin{array}{l}\text { Citation: Rahmati A, Farazi M, Choopanian F, Tahamtan M, Dashtelei A, Farhangdoosth H. Comparison of Inhibitory Con- } \\
\text { trol Skill in } 6 \text { to 8-Year-Old Children With Fluent Speech and Developmental Stuttering. Journal of Modern Rehabilitation. } \\
\text { 2018; 12(4):233-238. http://dx.doi.org/10.32598/JMR.V12.N4.233 }\end{array}$ \\
\hline 口ifi" & d.Ij http://dx.doi.org/10.32598/JMR.V12.N4.233 \\
\hline
\end{tabular}

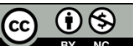

Article info:

Received: 23 Mar 2018

Accepted: 21 Aug 2018

Available Online: 01 Oct 2018

\section{Keywords:}

Inhibition, Children,

Developmental stuttering, Go/

No-Go task

\section{ABSTRACT}

Introduction: Fluent speech requires inhibitory control for the modification of speech interruptions before the onset or during the speech. The current study aimed at comparing the inhibitory control skill in 6 to 8 -year-old children with fluent speech and developmental stuttering.

Materials and Methods: This research is a descriptive-analytic cross-sectional study. The participants included 31 children with developmental stuttering (6-8 years old) selected based on the convenience sampling method. Also, 32 healthy children were selected from elementary schools in Tehran Province that matched in terms of age and gender with the first group. The children of two groups were selected from the same district (The fifth district) of Tehran Province. Visual Go/No-Go task was used to measure inhibitory control. The Mann-Whitney U test was used to compare the groups.

Results: The result showed that the mean score of children with stuttering in the inhibition index was lower than normal children. According to the Kolmogorov-Smirnov test, the data distribution was not normal $(\mathrm{P}=0.042)$. Therefore, the parametric test of the MannWhitney U test was used. Despite the lower mean score of inhibition in children with stuttering than healthy children, there was no significant difference in the inhibition index between the groups $(\mathrm{P}=0.550)$.

Conclusion: The findings indicated that Children Who Stutter (CWS) had less efficiency in inhibitory control skill compared to normal children, and this problem could be because of the exacerbation or persistence of stuttering.

* Corresponding Author:

Morteza Farazi, PhD.

Address: Department of Speech Therapy, University of Social Welfare and Rehabilitation Sciences, Tehran, Iran.

Tel: +98 (912) 1405628

E-mail: mfarazi80@gmail.com 


\section{Introduction}

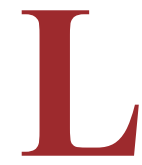

anguage is one of the cognitive functions of the human mind that is expressed in the form of speech. Speech requires a series of fine and coordinated activities. Interference in the performance of each of these activities leads to speech impairment. Disfluency is one of the speech disorders that appears in two forms of normal and abnormal or stuttering. Stuttering is a disorder often characterized by the interruptions of speech, including repetitions and prolongation of sounds, syllables, words, blocking in the flow of speech, interjections, and speech revision [1-3]. Stuttering consists of core and secondary behaviors. Core behaviors include repetition, prolongation, and blocking. Secondary behaviors comprised two categories of escape and avoidance behaviors [3]. Stuttering is divided into 3 groups based on its characteristics and causes; developmental, neurogenic, and psychogenic stuttering. Developmental stuttering is the most common type of stuttering that usually begins in childhood between the ages of 2 and 5 years. In the absence of self-improvement or lack of definitive treatment, developmental stuttering often persists and converts to the advanced or severe stuttering over time $[1,4,5]$.

Despite the great attention to stuttering, among other speech and language disorders, its etiology has not been well understood so far [6]. In the current study, stuttering is examined with a neuropsychological perspective to realize the different aspects of the involvement of neuropsychology in stuttering.

Various theories have been proposed regarding the origin of stuttering. Some researchers believe that stuttering results from interactions between a vulnerable speech motor system and many other factors such as genetic predisposition, emotional regulation, and linguistic and cognitive skills $[1,7]$.

Research has indicated that most children who stutter are different from their fluent peers, in various domains like cognitive functions. One of the cognitive processes is the executive function that refers to the higher-level cognitive functions involved in controlling and regulating lower-level cognitive processes and target-oriented behaviors that lead to the conscious control of thought and action [8-12].

Executive functions involve several cognitive processes, including inhibitory control, attentional shifting, planning, and working memory [13]. This study focuses on inhibitory control. Regarding the role of executive functions, each person before the onset or during the fluent speech requires identification, inhibition, and modification of speech interruptions (such as repetitions and prolongations), and must be able to consistently regulate the speech process to adjust the verbal units fluently and sequentially. Therefore, any defect in the inhibition, transmission, and working memory of the developing children can make speech fluency difficult [14]. Inhibitory control refers to the ability to suppress and postpone an inappropriate response in uncertain and new situations. This process is essential for daily activities and playing a role in cognitive development, executive function, and the conscious use of attention $[15,16]$.

Eggers et al. reported the difference between children with stuttering and normal peers in the context of inhibitory control based on a parenting questionnaire, using a computer task. The results indicated that compared to normal peers, children with stuttering had more false responses, less response time to the false ones, and less able to correct them after understanding the errors [15].

Regarding some of the pathological theories that consider cognitive factors effective in causing the stuttering, the central questions of the present research are whether the ability to control the inhibition is different between children with and normal peers, and whether to consider the lack of inhibitory control skill as a deficiency in children with stuttering. Therefore, we decided to examine the inhibitory control skill to help early cognitive interventions in these children and provide therapists with more effective strategies for evaluating and treating stuttering.

\section{Participants and Methods}

\section{Participants}

This research is a descriptive-analytic, cross-sectional study. The study population consisted of 6- to 8-year-old Persian-speaking children with fluent speech and developmental stuttering of Tehran Province. According to the sample size formula, 31 children with stuttering aged 6 to 8 years were recruited. They were selected, using convenience sampling method from speech and language therapy clinics and hospitals in Tehran. Also, 32 normal children (matched on age, sex, and handedness with the first group) were selected from the elementary schools of Tehran Province.

The inclusion criteria included: A. being at the beginning of stuttering during preschool or children with de- 
velopmental stuttering (research-based questionnaire); B. being 6 to 8 years old; C. lacking speech and language disorders such as cluttering, dysarthria, genetic syndromes, articulation disorder, etc. (research-based questionnaire); D. being monolingual Persian-speaking (research-based questionnaire); E. being right-handedness (informal assessment by the examiner); and f. having average IQ, hearing, and vision for performing the tasks (based on the child history). The exclusion criterion was the unwillingness of the participant to continue the cooperation at any point of the study.

\section{Study procedure}

The parents and supervisors of the health centers/ schools provided their consent before doing the tasks. Then, authors designed and used a questionnaire to record the demographic data, school grade, hearing status, visual status, general condition, laterality, language, history of stuttering in the family, stuttering onset age, other speech and language disorders, and medical history. Visual Go/No-Go task was used to measure inhibitory control.

Donders designed and standardized the visual Go/NoGo task for the first time in 1969 to measure the inhibition [17]. In this task, a few rectangular pairs with colors "white and blue" and "white and yellow" appear randomly on the monitor for a short time. The participant should carefully pay attention to these pairs of rectangles. If one of the displayed pairs is yellow, no response is required by the participant (Figure 1A). If one of the pairs is blue, two different conditions happen and, therefore, require two different responses. If the blue rectangular is on the right and the white is on the left, the participant should press the "question mark" button "?" on the keyboard with the maximum speed (Figure 1B), and if the white rectangular is on the right side and the blue is on the left, the participant should press the " $Z$ " button as fast as possible (Figure 1C).

Children's ability to perform this task improves as age increases. Therefore, the performance of the 8-year-old children is better than that of the 7-year-old children, and the performance of the 7-year-old children is better than that of the 6-year-olds. Before the implementation of the main phase of this task, a pilot phase was performed to prepare them by themselves [3]. Before doing the Go/No-Go task, it was performed for the subjects to educate them. There were 40 subtests, and the whole task considering the experimental stage, time of presentation, and the distance between the stimuli took about 20-25 minutes.

Before performing the main phase of the Go/No-Go task, the researchers taught participants how to do it, and asked them to try the task as a pilot phase to be prepared for the primary test. Go/No Go test consists of $40 \mathrm{sub}$ tests and takes about 20-25 minutes.

The Kolmogorov-Smirnov test (K-S test) was used to assess the normal distribution of the data. Also, because of the abnormal distribution of data $(\mathrm{P}<0.05)$, for the comparison of the Go/No-Go task, and mean score between two groups, non-parametric test of MannWhitney U test was used. The K-S test was used to assess the normal distribution of data. After realizing the abnormal distribution of data $(\mathrm{P}<0.05)$, the authors used the Mann-Whitney U test to compare the mean scores of the Go/No-Go test between the groups. The data were analyzed in SPSS V. 16.

\section{Results}

In this study, 31 children with stuttering (Mean \pm SD age of $6.87 \pm 0.83 \mathrm{y}$ ) and 32 children with normal speech (Mean \pm SD age of $6.93 \pm 0.81 \mathrm{y}$ ) were studied. The participants included 8 girls with stuttering, 8 girls with normal speech, 23 boys with stuttering, and 24 boys with normal speech. (Tables $1 \& 2$ ) present the descriptive analysis and mean scores of the Go/No-Go task in both groups.

As indicated in Table 1, the mean score of inhibition index in children with stuttering was lower than normal
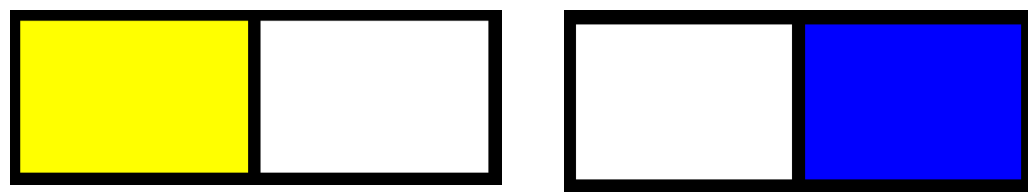

C

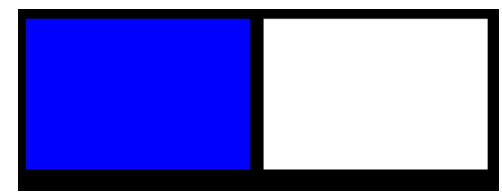

Figure 1. Overview of the Go/NoGo task

A. No response if one of the rectangles is yellow; B. Press "?" if one of the rectangles is blue and on the right side; C. Press " $Z$ " if one of the rectangles is blue and on the left side 
Table 1. Descriptive analysis of inhibition index of Go/No-Go task (N=63)

\begin{tabular}{llccc}
\hline Go/No-Go Task Index & Group & Mean \pm SD & Max & Min \\
\hline \multirow{2}{*}{ Inhibition } & Stuttering & $28.00 \pm 6.91$ & 38 & 13 \\
& Normal & $29.28 \pm 7.32$ & 40 & 12 \\
\hline
\end{tabular}

$J \mathrm{MR}$

Table 2. Mann-Whitney U test to compare the mean scores of inhibition index of Go/No-Go task

\begin{tabular}{ccccc}
\hline Index & Group & Number & Mean Score & Significance Level \\
\hline \multirow{2}{*}{ Inhibition } & Stuttering & 31 & 30.42 & 0.550 \\
& Normal & 32 & 33.53 & \\
\hline
\end{tabular}

children. According to the K-S test, the data distribution was not normal $(\mathrm{P}=0.042)$. Therefore, the Mann-Whitney $U$ test was used. Despite the lower mean score of inhibition index in children with stuttering, there was no significant difference in the inhibition index between the two groups $(\mathrm{P}=0.550)$.

\section{Discussion}

The current study aimed at comparing the inhibitory control skill between children with and without stuttering, using the Go/No Go task. Proper inhibitory control skill causes a delay in inappropriate responses in new situations and, hence, prevents inappropriate behaviors. Inhibitory control skill is necessary for daily activities and plays a role in cognitive development, executive function, and the conscious use of attention or attention control. Therefore, as there are errors in the normal speech flow of children with stuttering, a lower mean score of inhibitory control is predictable in children with stuttering compared to normal peers [14]. The main finding of the current study indicates that the mean score of inhibitory control index is lower than normal children, although there is no significant difference between both groups.

The results are in agreement with previous findings reported by Esfandeh et al. and Eggers et al. who reported lower inhibitory control in children with stuttering and no significant difference between the experimental and control groups $[15,18]$. Esfandeh et al. used Stroop color test to measure the inhibition in the primary school children, which is different from the current study from two aspects: the evaluation test and the age range of the study groups. Eggers et al. also studied primary school children.
The differences between the study of Esfande et al. and the current study are the type of assessment test and the age range of the participants. They used the Stroop color test to measure the inhibition in primary school children. Eggers et al. also studied primary school children.

The difference of inhibition index between the groups can be discussed based on the imaging studies. Brain imaging data indicated that right frontal cortex is one of the main anatomical connections of the inhibitory control. In addition to the frontal lobe, basal ganglia plays an essential role in the dominant right hemisphere network for inhibition [19]. Furthermore, imaging studies have revealed abnormal activities in these cortical and subcortical structures in individuals with stuttering. These structures constitute a part of a cortical-basal ganglia network that engages in conceptualizing the primary processes of developmental stuttering [20]. Considering the active brain areas in inhibitory control, and the existent damage to these areas (cortical and subcortical structures) in children with stuttering, the difference in the function of two groups can be explained.

\section{Conclusion}

The results of this study showed that although there is no significant difference between the experimental and control groups, children with stuttering showed less effectiveness in inhibitory control skill. This weakness may lead to the persistence or exacerbation of stuttering in them. Moreover, cognitive skills are weak in children with stuttering. 


\section{Ethical Considerations}

Compliance with ethical guidelines

The study was approved by the Ethics Committee of the University of Social Welfare and Rehabilitation Sciences.

Funding

This article is a extracted from the MSc. thesis of the first author, Department of Speech Therapy, University of Social Welfare and Rehabilitation Sciences, that has been approved at the University of Social Welfare and Rehabilitation Sciences, Tehran.

\section{Authors contributions}

Conceptualization: Atefeh Rahmati, Morteza Farazi, Hashem Farhangdoosth; Writing-original draft: Atefeh Rahmati; Investigation, resources, data curation: Atefeh Rahmati, Farhad Choopanian; Software, formal analysis: Aliakbar Dashtelei; Writing-review \& editing: Morteza Farazi, Mahdi Tahamtan.

\section{Conflict of interest}

The authors declared no conflict of interest.

Acknowledgements

The authors are grateful to the CEOs of the health centers for their kind help with conducting this study.

\section{References}

[1] Guitar B. Stuttering: An integrated approach to its nature and treatment. Philadelphia: Lippincott Williams \& Wilkins; 2013.

[2] Büchel C, Sommer M. What causes stuttering? PLOS Biology. 2004; 2(2):e46. [DOI:10.1371/journal.pbio.0020046] [PMID] [PMCID]

[3] Howell P. Signs of developmental stuttering up to age eight and at 12 plus. Clinical Psychology Review. 2007; 27(3):287-306. [DOI:10.1016/j.cpr.2006.08.005] [PMID] [PMCID]

[4] Craig A, Hancock K, Tran Y, Craig M, Peters K. Epidemiology of stuttering in the community across the entire life span. Journal of Speech, Language, and Hearing Research. 2002; 45(6):1097-105. [DOI:10.1044/1092-4388(2002/088)]

[5] Ward D. Stuttering and cluttering: Frameworks for understanding and treatment. London: Psychology Press; 2017.

[6] Smits-Bandstra S, De Nil L, Rochon E. The transition to increased automaticity during finger sequence learning in adult males who stutter. Journal of Fluency Disorders. 2006; 31(1):2242. [DOI:10.1016/j.jfludis.2005.11.004] [PMID]

[7] Felsenfeld S, van Beijsterveldt CEM, Boomsma DI. Attentional regulation in young twins with probable stuttering, high nonfluency, and typical fluency. Journal of Speech, Language, and Hearing Research. 2010; 53(5):1147-66. [DOI:10.1044/10924388(2010/09-0164)]

[8] Caruso AJ, Chodzko-Zajko WJ, Bidinger DA, Sommers RK. Adults who stutterresponses to cognitive stress. Journal of Speech, Language, and Hearing Research. 1994; 37(4):746-54. [DOI:10.1044/jshr.3704.746]

[9] Zelazo PD, Carter A, Reznick JS, Frye D. Early development of executive function: A problem-solving framework. Review of General Psychology. 1997; 1(2):198-226. [DOI:10.1037/10892680.1.2.198]

[10] Alvarez JA, Emory E. Executive function and the frontal lobes: A meta-analytic review. Neuropsychology Review. 2006; 16(1):17-42. [DOI:10.1007/s11065-006-9002-x] [PMID]

[11] Bialystok E, Viswanathan M. Components of executive control with advantages for bilingual children in two cultures. Cognition. 2009; 112(3):494-500. [DOI:10.1016/j.cognition.2009.06.014] [PMID] [PMCID]

[12] Best JR, Miller PH. A developmental perspective on executive function. Child Development. 2010; 81(6):1641-60. [DOI:10.1111/j.1467-8624.2010.01499.x] [PMID] [PMCID]

[13] Vithlani PP, Calkins SD. Emotion regulation and executive functioning as predictors of theory of mind competence during early childhood. Greensboro: University of North Carolina at Greensboro; 2010.

[14] Ahlsén E. Introduction to neurolinguistics. John Benjamins Publishing; 2006.

[15] Eggers K, Luc F, Van den Bergh BR. Inhibitory control in childhood stuttering. Journal of Fluency Disorders. 2013; 38(1):1-13. [DOI:10.1016/j.jfludis.2012.10.001] [PMID]

[16] Ntourou K, Anderson JD, Wagovich SA. Executive function and childhood stuttering: Parent ratings and evidence from a behavioral task. Journal of Fluency Disorders. 2018; 56:18-32. [DOI:10.1016/j.jfludis.2017.12.001] [PMID] [PMCID]

[17] Donders FC. On the speed of mental processes. Acta Psychologica. 1969; 30(1):412-31. [DOI:10.1016/0001-6918(69)90065-1]

[18] Esfandeh K, Ghorbani A, Farhangdoosth H, Nazari MA, Jalaie S. Comparison of shifting attention function in 7-13-yearsold children with fluent speech and developmental stuttering. Auditory and Vestibular Research. 2017; 23(1):70-8.

[19] Chambers CD, Garavan H, Bellgrove MA. Insights into the neural basis of response inhibition from cognitive and clinical neuroscience. Neuroscience \& Biobehavioral Reviews. 2009; 33(5):631-46. [DOI:10.1016/j.neubiorev.2008.08.016] [PMID]

[20] Alm PA. Stuttering and the basal ganglia circuits: A critical review of possible relations. Journal of Communication Disorders. 2004; 37(4):325-69. [DOI:10.1016/j.jcomdis.2004.03.001] [PMID] 
This Page Intentionally Left Blank 